\title{
Relativistic Equations of State at Finite Temperature
}

\author{
A. M. S. Santos and D. P. Menezes \\ Departamento de Física, CFM, Universidade Federal de Santa Catarina, \\ Florianópolis, SC, CP. 476, CEP 88.040-900, Brazil
}

Received on 29 September, 2003

\begin{abstract}
In this work we study the effects of temperature on the equations of state within a relativistic model, with the inclusion of the baryons of the octet over a wide range of densities. We compare the results of the equation of state, effective mass and strangeness fraction for the TM1, NL3 and GL sets of parameters.
\end{abstract}

\section{Motivation}

A neutron star is born after the gravitational collapse of the core of a very massive star. We briefly summarize the evolution process as follows: when massive stars in late stages of evolution (supergiants) come to a stage that thermal energy no longer bears the weight of the outer layers, gravity attracts the denser regions to the centre of the supergiant, and since thermal pressure is lower and lower as it cools, gravitational collapse comes next. Now with a denser and hotter core, the star starts fusing elements (beginning with hydrogen into helium, at $\mathrm{T} \sim 10^{7} \mathrm{~K}$ ), spending most of its life in this stage until the core contracts to form a white dwarf. Inverse beta decay lowers the high pressure provided by the relativistic eletrons in the core and it collapses. This core implosion takes about 0.5 seconds. The infalling material rebounce on the core, meets the decompression wave and ejects all but the core which cools, comes to an equilibrium composition of neutrons, protons, hyperons and leptons and is then called a neutron star. In [1] the authors study the equations of state (EOS) for cold neutron stars in a ThomasFermi framework, with the presence of the baryonic octet. In this work we study the effects of finite temperature on the EOS considering the presence of the hyperons of the octet. We work in the framework of the relativistic nonlinear
Walecka model (NLWM) [2], where hadrons are coupled to scalar-isoscalar $\phi$, vector-isoscalar $V^{\mu}$ and vector isovector $\vec{b}^{\mu}$ meson fields. Temperature effects are taken into account by including the Thomas-Fermi distribution function in the EOS. Neutron stars are not made up of neutrons only. At neutron star densities, although satisfying charge neutrality, pure neutron matter is not the lowest energy state. For this reason we include in the treatment the leptons $e^{-}$ and $\mu^{-}$, as fermi gases of noninteracting particles. We then carry out a comparative study of the different results of the NLWM in the context of some different sets of parameters, namely: TM1 [3], GL [4] and NL3 [5]. We study the behaviour of the effective mass curves of baryons with these three parameter sets at different temperatures and densities, whilst performing the calculations through a wide range of baryonic densities (at least up to $\sim 6.0$ times the saturation density). We also investigate the strangeness fraction at finite temperature for the parameter sets used in this work and finally draw our conclusions.

\section{The NLWM at Finite Temperature}

The Lagrangian density in the model reads:

$$
\begin{aligned}
\mathcal{L} & =\sum_{B} \overline{\psi_{B}}\left[\gamma_{\mu}\left(i \partial^{\mu}-g_{v B} V^{\mu}-\frac{g_{\rho B}}{2} \gamma_{\mu} \vec{\tau} \cdot \mathbf{b}^{\mu}\right)-\mathbf{M}_{\mathbf{B}}^{*}\right] \psi_{B} \\
& +\frac{1}{2}\left(\partial_{\mu} \phi \partial^{\mu} \phi-m_{s}^{2} \phi^{2}\right)-\frac{1}{3 !} \kappa \phi^{3}-\frac{1}{4 !} \lambda \phi^{4}-\frac{1}{4} \Omega_{\mu \nu} \Omega^{\mu \nu} \\
& +\frac{1}{2} m_{v}^{2} V_{\mu} V^{\mu}+\frac{1}{4 !} \xi g_{v}^{4}\left(V_{\mu} V^{\mu}\right)^{2}-\frac{1}{4} \mathbf{B}_{\mu \nu} \cdot \mathbf{B}^{\mu \nu}+\frac{\mathbf{1}}{\mathbf{2}} \mathbf{m}_{\rho}^{2} \mathbf{b}_{\mu} \cdot \mathbf{b}^{\mu} \\
& +\sum_{l=e^{-}, \mu^{-}} \overline{\psi_{l}}\left(i \gamma_{\mu} \partial^{\mu}-m_{l}\right) \psi_{l},
\end{aligned}
$$

where

$\Omega_{\mu \nu}=\partial_{\mu} V_{\nu}-\partial_{\nu} V_{\mu}, \quad \mathbf{B}_{\mu \nu}=\partial_{\mu} \mathbf{b}_{\nu}-\partial_{\nu} \mathbf{b}_{\mu}-\mathbf{g}_{\rho}\left(\mathbf{b}_{\mu} \times \mathbf{b}_{\nu}\right)$,

and $g_{\nu B}=\chi_{v B} g_{v}, g_{\rho B}=\chi_{\rho B} g_{\rho}$ and $g_{s B}=\chi_{s B} g_{s}$ are the coupling constants of baryon " $B$ " to the mesons; $M_{B}{ }^{*}=M_{B}-g_{s B} \phi, M_{B}$ is the mass of baryon " $B$ ", $m_{v}$, $m_{\rho}$ and $m_{s}$ are the masses of the mesons.

From the Euler-Lagrange formalism we obtain the equa- 
tions of motion, replacing the meson fields by their mean values (relativistic mean-field approximation). The equations of motion reduce to:

$$
\begin{gathered}
\phi_{0}=-\frac{k}{2 m_{s}^{2}} \phi_{0}^{2}-\frac{\lambda}{6 m_{s}^{2}} \phi_{0}^{3}+\sum_{B} \frac{g_{s}}{m_{s}^{2}} \chi_{s B} \rho_{s B} \\
V_{0}=-\frac{\xi}{6 m_{v}^{2}} g_{v}^{4}\left(V_{0}\right)^{3}+\sum_{B} \frac{g_{v}}{m_{v}^{2}} \chi_{v B} \rho_{B} \\
\mathbf{b}_{0}=\sum_{B} \frac{g_{\rho}}{m_{\rho}^{2}} \chi_{\rho B} \tau_{3 B} \rho_{B},
\end{gathered}
$$

where

$$
\begin{gathered}
\rho_{s B}=\frac{1}{\pi^{2}} \int p^{2} d p \frac{M_{B}^{*}}{\epsilon_{B}}\left(f_{B+}+f_{B-}\right), \\
\rho_{B}=\frac{1}{\pi^{2}} \int p^{2} d p\left(f_{B+}-f_{B-}\right)
\end{gathered}
$$

and $\tau_{3 B}$ is the appropriate isospin projector for the baryon charge states. The distribuition function for the baryons is

$$
f_{B \pm}=\frac{1}{1+e^{\left[\left(\epsilon_{B} \mp \nu_{B 0}\right) / T\right]}} .
$$

In the static, homogeneous nuclear matter assumption we have made, the terms $\epsilon_{B}$ and $\nu_{B 0}$ are given respectively by $\epsilon_{B}=\sqrt{p^{2}+M_{B}^{* 2}}$ and $\nu_{B 0}=\mu_{B}-g_{\nu B} V_{0}-g_{\rho B} \tau_{3 B} b_{0}$.

Since the star has come to equilibrium, particle populations have come to a stationary number where the total electric charge is neutral and chemical equilibrium is required: $\mu_{\Sigma^{0}}=\mu_{\Xi^{0}}=\mu_{\Lambda}=\mu_{n}, \mu_{\Sigma^{-}}=\mu_{\Xi^{-}}=\mu_{n+} \mu_{e^{-}}$, $\mu_{\Sigma^{+}}=\mu_{p}=\mu_{n}-\mu_{e^{-}}$.

The expressions for the energy density in this model at finite temperature reads:

$$
\begin{aligned}
\mathcal{E}_{T} & =\frac{1}{\pi^{2}} \sum_{B} \int p^{2} d p \sqrt{p^{2}+M_{B}^{* 2}}\left(f_{B+}+f_{B-}\right) \\
& +\frac{1}{\pi^{2}} \sum_{l} \int p^{2} d p \sqrt{p^{2}+m_{l}^{2}}\left(f_{l-}+f_{l+}\right)+\frac{m_{v}^{2}}{2} V_{0}^{2} \\
& +\frac{\xi g_{v}^{4}}{8} V_{0}^{4}+\frac{m_{\rho}^{2}}{2} b_{0}^{2}+\frac{m_{s}^{2}}{2} \phi_{0}^{2}+\frac{\kappa}{6} \phi_{0}^{3}+\frac{\lambda}{24} \phi_{0}^{4},
\end{aligned}
$$

where the distribuition function for the leptons and antileptons are:

$$
f_{l \pm}=\frac{1}{1+e^{\left[\left(\epsilon_{l} \mp \mu_{l}\right) / T\right]}} .
$$

where $\epsilon_{l}=\sqrt{p_{l}^{2}+m_{l}^{2}}$ and $\mu_{l}$ is the chemical potential of lepton "l". The expression for the pressure can also be obtained in a straightforward manner.

\section{A comparative study of the TM1, GL and NL3 sets of parameters}

We show now our results for the EOS, effective mass, particle populations and strangeness fraction in the NLWM with the three sets of parameters shown in table I.

The EOS for the three sets are shown in Fig. 1. At zero temperature, we see that GL and NL3 provide us with the same results up to $3.5 \rho / \rho_{0}$, where the EOS for NL3 collapses, while TM1 gives a smoother curve. At $10 \mathrm{MeV}$, the GL EOS becomes slightly harder than the NL3 curve. In all cases the energy density values increase with temperature.

From the values of effective masses given in Fig. 2 we conclude that GL provides a satisfactory description of the nuclear matter for a wide range of densities; TM1 fails to describe the effective mass if hyperons are included, as the baryonic density comes to $\sim 6.5$ times the nuclear saturation density; NL3 also fails at $\sim 3.5$ times the saturation density. The curves for higher temperatures are virtually the same as for $\mathrm{T}=0$, so we do not show them here. Notice that TM1 and NL3 only fail because baryons other than protons and neutrons were included.

From Fig. 3 we see that electrical neutrality is conserved: positively charged particles have their population increased as negative hyperons appear. Heaviest hyperons come about at higher densities, as expected - inner regions of neutron stars are presumably populated by heavier baryons. We see in Fig. 4 the particle populations for higher temperatures. Hyperons turn up at lower densities as temperature increases and more hyperons show up. The heaviest baryon of the octet $\Xi^{0}$ also appears. All plots of particle population that we show here have been obtained with the TM1 set. For densities higher than $6.5 \rho / \rho_{0}$, only the GL parametriztion can be used.

Concerning the strangeness fraction shown in Fig. 5, different parameter sets provide different results for its values. The strangeness fraction reaches almost $1 / 3$ for the GL parametrization at $10 \rho / \rho_{0}$ (density of the interior of a protoneutron star). TM1 and NL3 provide remarkably higher values of strangeness fraction for lower densities. For all three sets the values have a slight increase with temperature. We conclude that strangeness fractions are more sensitive to the parametrization than to temperature.

TABLE 1.The table shows the values for the parameters. All masses are given in Mev.

\begin{tabular}{ccccccccccc}
\hline \hline Force & $M$ & $m_{s}$ & $m_{v}$ & $m_{\rho}$ & $g_{s}$ & $g_{v}$ & $g_{\rho}$ & $\kappa / M$ & $\lambda$ & $\xi$ \\
\hline TM1 & 938.0 & 511.198 & 783.0 & 770.0 & 10.0289 & 12.6139 & 9.2644 & 3.04 & 3.7098 & 0.0169 \\
\hline NL3 & 939.0 & 508.194 & 782.501 & 763.0 & 10.217 & 12.868 & 8.948 & 4.377 & -173.31 & 0.0 \\
\hline GL & 938.0 & 511.198 & 783.0 & 770.0 & 8.175 & 9.182 & 9.717 & 4.524 & 191.864 & 0.0 \\
\hline \hline
\end{tabular}




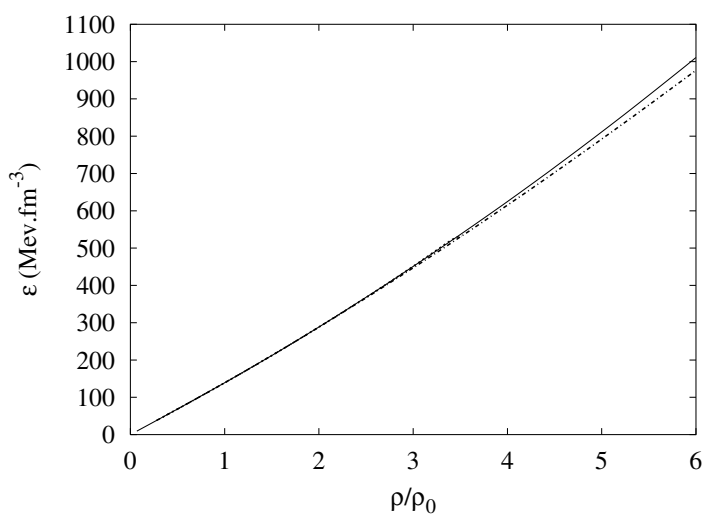

a)

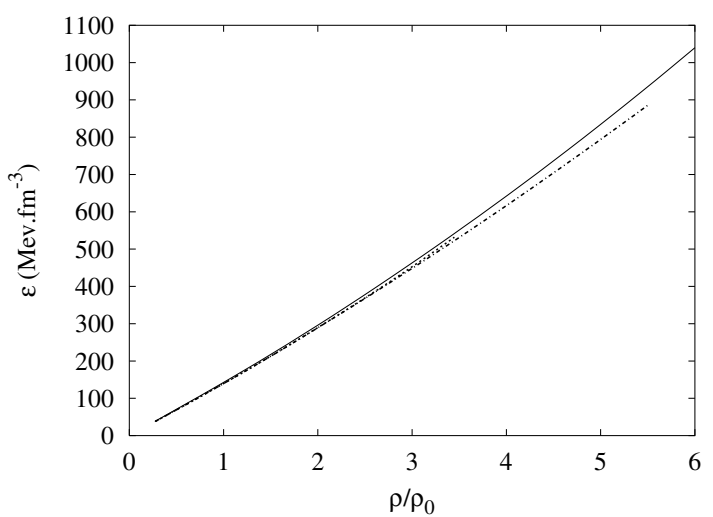

b)

Figure 1. Energy density in the NLWM in the presence of the octet, at a)T=0, b) 10 Mev with different sets, namely: GL (solid line), NL3 (dashed line) and TM1 (dot-dashed line). At zero temperature the curves with GL and NL3 are coincident.

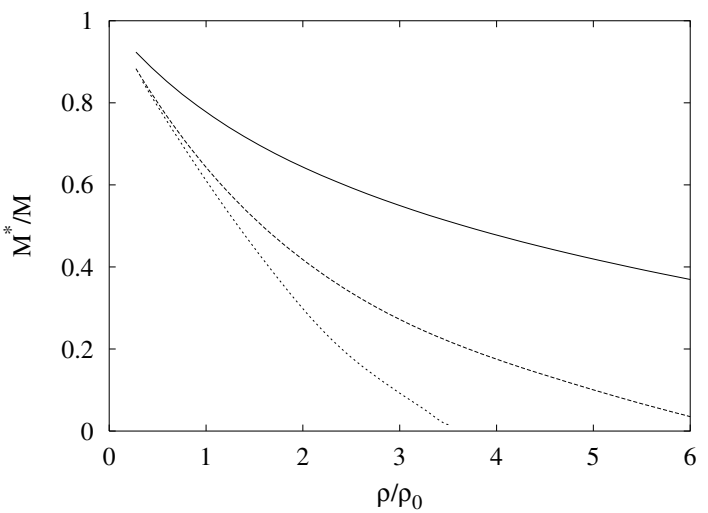

Figure 2. Effective mass curves for nucleons in the NLWM model, with parametrizations NL3 (bottom), TM1 and GL (top), at zero temperature.

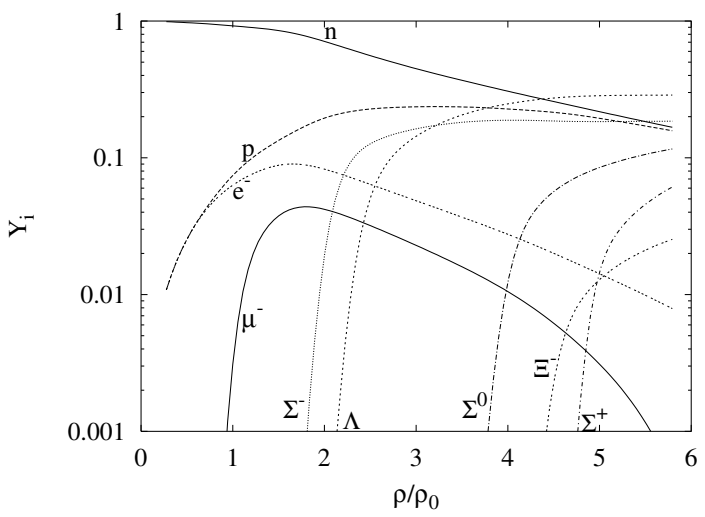

Figure 3. Particle population $Y_{i}=\rho_{i} / \rho_{B}, i=$ baryons and leptons at zero temperature. 


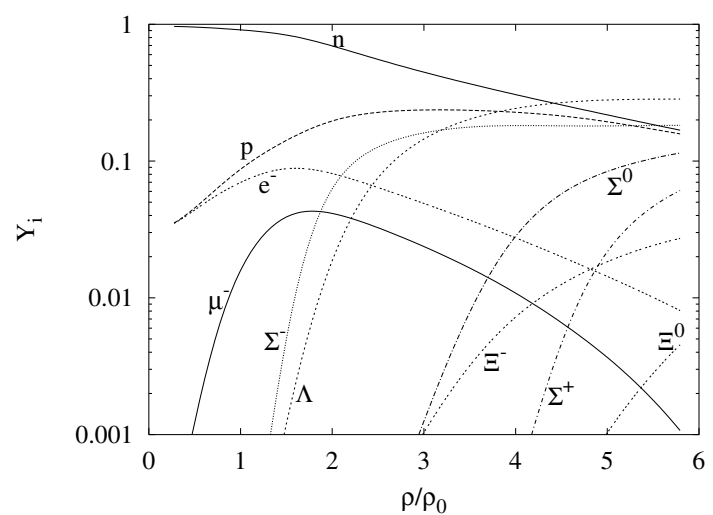

a)

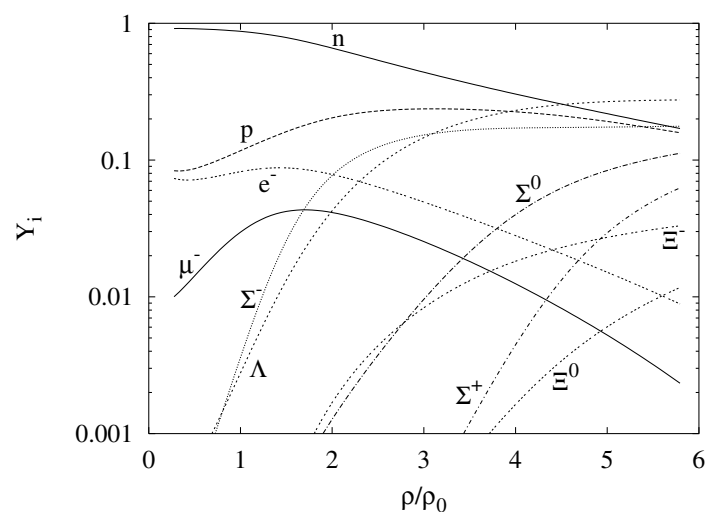

b)

Figure 4. The same as in Fig. 3 for a)T=10 MeV, b)T=20 MeV.

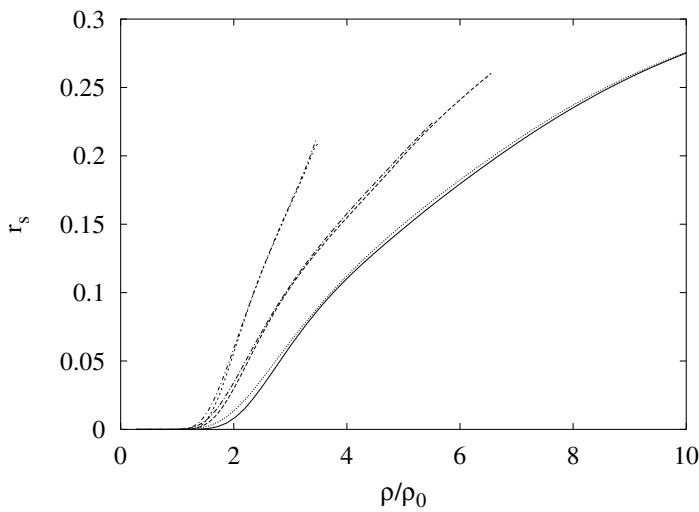

Figure 5. Strangeness fractions at 10 and $20 \mathrm{MeV}$ for the three sets of parameters. Left curves for NL3, TM1 in the center and GL in the right hand side. The lower values for each parametrization stand for the EOS in the NLWM at $10 \mathrm{MeV}$, higher values for $20 \mathrm{MeV}$.

One of the authors (A.M.S. Santos) would like to thank Dr. Prafulla Panda and Dr. Constança Providência for their suggestions and comments on this work.

\section{References}

[1] A. L. Espíndola and D. P. Menezes, Phys. Rev. C 65, 045803 (2002)
[2] B. D. Serot and J. D. Walecka. Adv. Nucl. Phys. 16 (1985) 1; J. Boguta and A. R. Bodmer, Nucl. Phys. A. 292 (1977) 41.

[3] K. Sumiyoshi, H. Kuwabara, and H. Toki, Nucl. Phys. A 581, 725 (1995).

[4] N.K. Glendennig, Astrophys. J. 293, 470 (1985).

[5] G. A. Lalazissis, J. König and P. Ring Phys. Rev. C 551 (1997). 